\title{
Full-length genomic and molecular characterization of Canine parvovirus in dogs from North of Brazil
}

\author{
S.P. Silva ${ }^{1}$, L.N.P.P. Silva ${ }^{1}$, E.D.L. Rodrigues ${ }^{2}$, J.F. Cardoso ${ }^{1}$, F.N. Tavares ${ }^{3}$, \\ W.M. Souza ${ }^{4}$, C.M.P. Santos², F.M.S. Martins², I.S. Jesus ${ }^{2}$, T.C. Brito ${ }^{2}$, \\ T.P.C. Moura ${ }^{2}$, M.R.T. Nunes ${ }^{1}$, L.M.N. Casseb ${ }^{3}$, E. Silva Filho ${ }^{2}$ and A.R. Casseb ${ }^{2}$ \\ ${ }^{1}$ Centro de Inovação Tecnológica, Instituto Evandro Chagas, \\ Ministério da Saúde, Ananindeua, PA, Brasil \\ ${ }^{2}$ Laboratório de Biologia Molecular, \\ Universidade Federal Rural da Amazônia, Belém, PA, Brasil \\ ${ }^{3}$ Departamento de Virologia, Instituto Evandro Chagas, Ananindeua, PA, Brasil \\ ${ }^{4}$ Centro de Pesquisa em Virologia, Faculdade de Medicina de Ribeirão Preto, \\ Universidade de São Paulo, Ribeirão Preto, SP, Brasil \\ Corresponding author: A.R. Casseb \\ E-mail: alexcasseb@yahoo.com.br
}

Genet. Mol. Res. 16 (3): gmr16039719

Received May 12, 2017

Accepted August 11, 2017

Published September 21, 2017

DOI http://dx.doi.org/10.4238/gmr16039719

Copyright (C) 2017 The Authors. This is an open-access article distributed under the terms of the Creative Commons Attribution ShareAlike (CC BY-SA) 4.0 License.

\begin{abstract}
With the objective of characterizing Canine parvovirus (CPV) from some suspected fecal samples of dogs collected from the Veterinarian Hospital in Belém city, five positive samples were found by PCR assay and an update molecular characterization was provided of the CPV-2 circulation in Belém. Through sequencing of the complete DNA sequences (NS1, NS2, VP1, and VP2 genes), the CPV-2 strain was identified as CPV-2b (Asn426Asp) circulating in Belém. The $\mathrm{CPV}-2 \mathrm{~b}$ strain with a different change at the position Tyr324Leu was detected in all samples assessed and thus reported for the first time for the scientific community. Phylogenetic analysis indicated that Belém CPV-2b and CPV-2a strains would be related to a cluster with samples
\end{abstract}


after the 1990s, suggesting that CPV-2b in Belém originated from CPV2a circulating in Brazil after the 1990s. Potential recombination events were analyzed using RDP4 and SplitsTree4; therefore, results suggest that $\mathrm{CPV}-2$ sequences here described were not potentially recombination events. Continuous monitoring and molecular characterization of CPV2 samples are needed not only to identify possible genetic and antigenic changes that may interfere with the effectiveness of vaccines but also to bring a better understanding of the mechanisms that drive the evolution of CPV-2 in Brazil.

Key words: CPV-2; Dog; PCR; Phylogenetic analysis; DNA virus

\section{INTRODUCTION}

The Canine parvovirus type 2 (CPV-2) was first isolated or described during 1978 as the cause of new diseases in dogs everywhere in the world, when it rapidly spread throughout domestic populations, as well as becoming widespread in wild dogs. However, testing for antibodies in the sera collected from dogs or related canids showed that the first positive titers were present in dogs in Europe between 1974 and 1976 (Parrish, 1999; Parrish et al., 1988). Currently, the original CPV-2 has been completely substituted by three major genetic and antigenic variants (Fan et al., 2016), termed as CPV-2a (Parrish et al., 1988), CPV-2b (Parrish et al., 1991), and CPV-2c (Buonavoglia et al., 2001), identified in 1980, 1984, and 2000, respectively.

The CPV-2 has a linear single-stranded DNA (ssDNA) genome about $5 \mathrm{~kb}$ in length with two major open reading frames (ORFs). They encode two gene cassettes: a non-structural replicase gene (NS1 and NS2) that encodes the enzymes required for replication, and a capsid (VP1 and VP2) gene encoding structural proteins (Shackelton et al., 2005). This CPV-2 is a variant of Carnivore protoparvovirus 1 that belongs to the genus Protoparvovirus, within of the Parvoviridae family. This species includes Feline panleukopenia virus, Mink enteritis virus, and Raccoon parvovirus, according to the International Committee on Taxonomy of Viruses (Cotmore et al., 2014). Although CPV is a DNA virus, previous reports demonstrated that the high rate of nucleotide substitution during CPV-2 replication seems to be similar to that reported for RNA viruses (Shackelton et al., 2005).

The CPV-2 is one of the most important enteric pathogens of dogs. This virus is extremely contagious causing high morbidity with increased incidence in shelters, pet stores, and breeding kennels (Miranda and Thompson, 2016). The etiology of hemorrhagic gastroenteritis is mainly attributed to the CPV-2 over other microorganisms, often in dogs younger than 6 months (Strottmann et al., 2008).

The objectives of this study were to detect and characterize CPV-2 in dogs from Belém city, Brazil, by molecular analyses. Besides the genetic divergence in the CPV-2 variants, the occurrence of recombination events was also estimated.

\section{MATERIAL AND METHODS}

\section{Ethics statement}

The use, handling, and disposal of biological materials used in this project are in

Genetics and Molecular Research 16 (3): gmr16039719 
agreement with the ethical principles for Animal Experimentation adopted by the National Council of Animal Experimentation Control (CONCEA) and was approved by the Ethics Committee of Animal Use of Instituto Evandro Chagas (CEUA/IEC; number 02/2016).

\section{Sample, ssDNA extraction, and PCR}

Six fecal samples were collected from dogs unvaccinated to CPV-2 with vomit and diarrhea symptoms from the Veterinary Hospital in Belém city, Brazil. The ssDNA was extracted using the QIAamp ${ }^{\circledR}$ Viral RNA Mini Kit and testing by PCR. The primers used for PCR detection of CPV-F (5'-GCCATTTACTCCAGCAGC-3') and CPV-R (5'-AGTAAGTGTACTGGCACAG-3') that amplified a fragment with 216 bp of the VP2 gene, as previously described (Cho et al., 2006). For the amplification, the following conditions were applied: 35 cycles of denaturation at $94^{\circ} \mathrm{C}$ for $30 \mathrm{~s}$, annealing at $55^{\circ} \mathrm{C}$ for $30 \mathrm{~s}$ and polymerization at $72^{\circ} \mathrm{C}$ for $40 \mathrm{~s}$, followed by electrophoresis on a $2 \%$ agarose gel with $\mathrm{SYBR}^{\circledR}$ Safe DNA Gel Stain. All steps included a positive control performed using the commercial vaccine, and water as negative control.

\section{Genome sequencing}

The second strand of the ssDNA synthesis was performed using the cDNA Synthesis System kit (Roche Diagnostics) and $400 \mu \mathrm{M}$ Roche Primer "random". The reaction was purified with Agencourt AMPure XP Reagent (Beckman Coulter). The CPV-2 genome was recovered using the pyrosequencing approach (Margulies et al., 2005). DNA library was prepared and sequencing on a GS FLX+ (Roche Diagnostics) at the Center for Technological Innovation of Instituto Evandro Chagas, Ministry of Health, Brazil.

\section{Genome assembly}

The assembly sequence was carried out using the GS De Novo Assembler program (Newbler v. 3.0). The inspection, annotations of putative ORF genes, and additional analysis were performed using the Geneious v. 9.1.6 software (Biomatters, New Zealand).

\section{Estimating selection pressures}

The non-synonymous:synonymous nucleotide change ratio per site $(\mathrm{dN}: \mathrm{dS})$ for the VP2 protein was determined. The $\mathrm{dN}$ :dS ratio was estimated using the Datamonkey web interface (http://www.datamonkey.org). The neighbor joining tree was selected to generate data for use in the analysis. The HKY85 nucleotide substitution model was chosen for this prediction. The method performed was single-likelihood ancestor counting to identify selection acting on each codon position in a protein sequence (Pond and Frost, 2005; Zhang et al., 2006).

\section{Phylogeny analysis}

A multiple sequence alignment (MSA) was performed using the Mafft v. 7 program (Katoh and Standley, 2013). Before phylogenetic analysis, the jModelTest was applied to select the best-fit models of nucleotide substitution (Darriba et al., 2012). The reconstruction

Genetics and Molecular Research 16 (3): gmr16039719 
of phylogenetic trees was performed using the maximum likelihood (ML) method (Myung, 2003) implemented in the RaxML v. 8.2.4 (Stamatakis, 2014). For determination of the reliability of the tree topology, bootstrap analysis (Felsenstein, 1985) was carried out in 1000 replicates.

\section{Evolution of genetic recombination}

To identify potential recombination events, an MSA with complete genome of CPV-2 was performed using the Mafft v. 7 program (Katoh and Standley, 2013). Potential recombination events were then analyzed using RDP, GENECONV, Bootscan, MaxChi, Chimaera, SiScan, and 3Seq methods implemented in RDP4 (Martin et al., 2015) and SplitsTree4 (Huson and Bryant, 2006). Standard program settings for all methods were used to perceive sequences as linear, to require phylogenetic evidence, to refine breakpoints, and to check alignment consistency. The highest acceptable $\mathrm{P}$ value was set at 0.05 , after considering Bonferroni correction for multiple comparisons. All method-specific program settings remained at their default values.

\section{RESULTS}

Five of the six dog fecal samples were amplified and confirmed the presence of CPV2 by PCR. Three positive samples in the PCR to CPV-2 were sequencing, and the full-length ORF sequences were recovered; these three samples were Bel2014-01 (4891 nt), Bel2015-01 (4818 nt), and Bel2015-02 (5020 nt). The nucleotide sequences identified were released in NCBI with accession numbers KX774249, KX774251, and KX774252, respectively. Sequence comparisons revealed 99.1 to $99.9 \%$ nucleotide identities within these three samples.

Based on an analysis of complete VP2 sequences of three CPV-2 samples, which have their genomes sequenced with a reference sequence (M38245), we observed that a total of eight substitutions of amino acid residues occurred in three CPV-2 samples reported here. To characterize the CPV-2 variants shed in dog feces, the possible nucleotide changes at residues 426 were performed on the VP2 gene, and the sequencing results revealed that all sequences showed aspartic acid (426Asp) in this position, which evidenced that all samples reported here were a CPV-2b strain (Table 1). Also, an additional genomic region in the VP2 protein showed amino acid substitutions in residues 87, 101, 297, 300, 305, 324, and 375.

Samples of CPV-2 isolated in Brazil before the 1990s have a serine residue at position 297 (297Ser). However, after the 1990s a substitution of serine to alanine (Ser297Ala) occurred, and we found the same alanine residue in this position (297Ala) in all samples sequenced. Only a CPV-2 strain reported in Brazil (DQ340430) has a different residue in this position (Ser297Asn).

The analysis method of the selection pressure driving the CPV-2 evolution showed a mean $\mathrm{dN}$ : $\mathrm{dS}$ ratio of 0.164 for the VP2 protein gene. Five codons $(13,324,375,426$, and 440) were detected, and positively selected sites were identified in CPV-2 sequences using the default significance level ( $\mathrm{P}$ value/Bayes factor/posterior probability) of 0.1 and eighty negative selection sites.

The phylogenetic tree based on nucleotide sequences of the VP2 gene shows that all sequences reported in this study were clustered in the same clade (Figure 1). In general, the resulting of the ML tree showed low values of nodes, not supported by high bootstrap values,

Genetics and Molecular Research 16 (3): gmr16039719 
showing that the VP2 gene is not highly informative from a phylogenetic analysis. However, phylogenetic tree reveals that Brazil samples resulted in two distinct clades before and after the 1990s, and samples reported here are grouped with CPV-2a isolated after the 1990s.

\section{Table 1. Amino acid variations of CPV-2b samples in the VP2 protein.}

\begin{tabular}{|c|c|c|c|c|c|c|c|c|c|c|c|}
\hline \multirow{2}{*}{ Accession No. } & \multirow{2}{*}{ Location } & \multirow{2}{*}{ Collection date } & \multirow{2}{*}{ CPV type } & \multicolumn{8}{|c|}{ Substitution of amino acid residues in VP2 protein of CPV } \\
\hline & & & & 87 (Leu) & $101(\mathrm{Thr})$ & 297 (Ala) & 300 (Gly) & 305 (Tyr) & $324(\mathrm{Tyr})$ & 375 (Asp) & 426 (Asn) \\
\hline GU569943 & China & 1983 & 2 & Met & Ile & Ser & Asp & Asp & - & Asn & - \\
\hline M38245 & USA & 1996 & 2 & Met & Ile & Ser & Ala & Asp & - & Asn & - \\
\hline FJ222824 & Italy & 2005 & 2 & Met & Ile & Ser & Ala & Asp & - & Asn & - \\
\hline DQ340404 & Brazil & 1980 & $2 \mathrm{a}$ & - & - & Ser & - & - & - & - & - \\
\hline M24003 & USA & 1984 & $2 a$ & - & - & Ser & - & - & - & - & - \\
\hline DQ340410 & Brazil & 1986 & $2 \mathrm{a}$ & - & - & Ser & - & - & - & - & - \\
\hline DQ340415 & Brazil & 1991 & $2 \mathrm{a}$ & - & - & - & - & - & - & - & - \\
\hline DQ340430 & Brazil & 1995 & $2 a$ & - & - & Asn & - & - & - & - & - \\
\hline DQ340434 & Brazil & 2000 & $2 a$ & - & - & - & - & - & - & - & - \\
\hline GQ379043 & Thailand & 2008 & $2 \mathrm{a}$ & - & - & - & - & - & Ile & - & - \\
\hline KR559896 & Portugal & 2012 & $2 a$ & - & - & - & - & - & - & - & - \\
\hline KF149976 & Ecuador & 2012 & $2 a$ & - & - & - & - & - & - & - & - \\
\hline M74849 & USA & 1984 & $2 b$ & - & - & Ser & - & - & - & - & Asp \\
\hline DQ340409 & Brazil & 1985 & $2 \mathrm{~b}$ & - & - & Ser & - & - & - & - & Asp \\
\hline JQ743893 & China & 2011 & $2 \mathrm{~b}$ & - & - & - & - & - & Ile & - & Asp \\
\hline KF149972 & Ecuador & 2012 & $2 b$ & - & - & - & - & - & - & - & Asp \\
\hline KR559892 & Portugal & 2012 & $2 \mathrm{~b}$ & - & - & - & - & - & - & - & Asp \\
\hline KX774249* & Brazil & 2014 & $2 \mathrm{~b}$ & - & - & - & - & - & Leu & - & Asp \\
\hline KX774251* & Brazil & 2015 & $2 \mathrm{~b}$ & - & - & - & - & - & Leu & - & Asp \\
\hline KX774252* & Brazil & 2015 & $2 \mathrm{~b}$ & - & - & - & - & - & Leu & - & Asp \\
\hline GU380303 & China & 2009 & $2 \mathrm{c}$ & - & - & - & - & - & Ile & - & Glu \\
\hline KR559894 & Portugal & 2012 & $2 \mathrm{c}$ & - & - & - & - & - & - & - & Glu \\
\hline KF149965 & Ecuador & 2012 & $2 \mathrm{c}$ & - & - & - & - & - & - & - & Glu \\
\hline KT162019 & China & 2014 & $2 \mathrm{c}$ & - & - & - & - & - & Ile & - & Glu \\
\hline
\end{tabular}

*Samples described in this study.

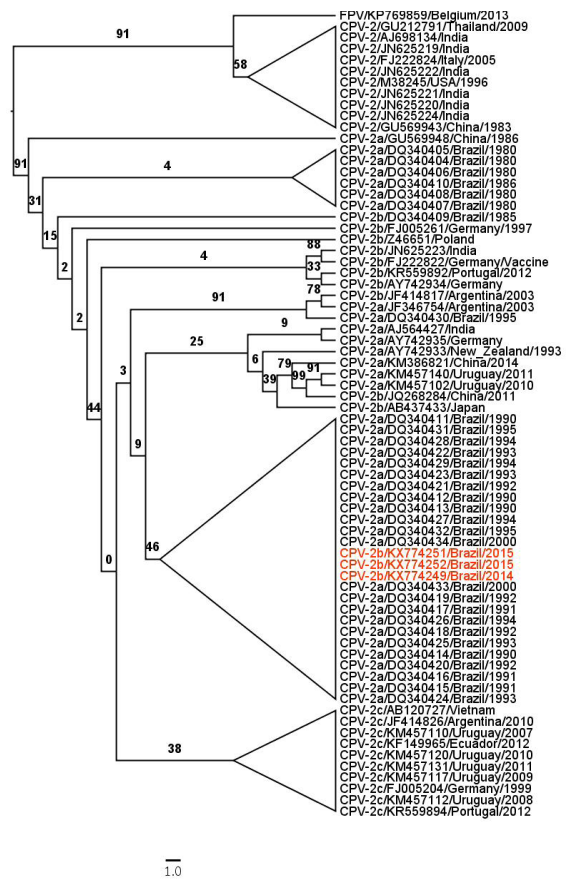

Figure 1. Phylogenetic analysis using the ML method of different samples within the species of Carnivore protoparvovirus 1 based on complete nucleotide sequences of the VP2 gene. Different phylogenetic groups are labeled in the different clade. Numbers over each main node of the tree correspond to bootstrap values (1000 replicates).

Genetics and Molecular Research 16 (3): gmr16039719 
Based on RDP4, a potential recombination event was identified in KX774251 (strain Bel2015-01), but only two methods, the Chimaera $\left(1.825 \times 10^{-2}\right)$ and 3Seq $(1.301 \mathrm{x}$ $10^{-3}$ ) suggested this potential recombinant event. Thus, to further analyze the recombination event, multiple sequence alignments were divided into two alignments based on the site of the breakpoint, and separate phylogenetic trees for each dataset were constructed (data not shown). The phylogenetic trees constructed with non-recombinant fragments of the NS and VP2 genes did not corroborate the chimeric pattern found in the genome of the KX774251. Also, the analysis in SplitsTree4 did not find potential reassortment events in CPV-2 sequenced in this study. Therefore, these results suggest that the CPV-2 sequences described here were not potentially recombination events, but future studies may help to clarify this point.

\section{DISCUSSION}

The original CPV-2 had previously been replaced between 1979 and 1981 by the CPV-2a strain through five/six amino acid mutations in the major capsid protein VP2 (residues Met87Leu, Ile101Thr, Ala300Gly, Asp305Tyr, and Asn375Asp) (Parrish et al., 1988). After 1986, most viruses isolated from dogs in many places of the USA were distinct antigenically from CPV-2a. The new antigenic type, the CPV-2b has largely replaced the previous antigenic type CPV-2a among virus isolates in this same place, because of a mutation in residue Asn426Asp in theVP2 protein (Parrish et al., 1991).

In this study, we reported the first full genome of CPV-2 in Brazil; previous studies have reported only the partial sequences from the VP2 gene. To characterize these CPV-2 samples, we showed the same non-synonymous mutation at amino acid residues 87, 101, 300, 305 , and 375 from CPV-2 to CPV-2a related. However, in the residue 426 of the VP2 protein, we found a substitution of Asn to Asp that characterizes the three samples of this study as the CPV-2b strain (Table 1). Thus, it is the first molecular characterization of CPV-2 related to strain $2 b$ in Belém city, North of Brazil.

The CPV-2 is epizootic in Brazil and followed the same pattern observed in the emergence of CPV-2 in the USA, which occurs with the replacement of variants CPV-2a to CPV-2b. In Brazil, the predominant strain reported in 1980 was CPV-2a, which was substantially replaced by CPV-2b from 1990 to 1995 (Pereira et al., 2000). Then, CPV-2c was the principal type that circulated in Brazil between the years 2008 and 2010 (Pinto et al., 2012). However, previous studies have described the distribution of CPV-2 in the south and southeast of Brazil, while we reported the first description of three samples of the CPV-2b strain in North of Brazil.

Previous studies by Shackelton et al. (2005) and Hoelzer et al. (2008) showed evidence for strong positive selection on the capsid VP2 protein gene with four VP2 residues in four sites $(101,232,300$, and 426) and $(101,300,324$, and 426), respectively. In this study, we found five positive selections in residues 13, 375, and 440, as well as in residues 324 and 426, as previously reported. On the other hand, Decaro et al. (2009) not detect any positively selected sites; probably this result was due to the different datasets used for inferring the selection pressure.

The residue 324 is adjacent to residue 323 , which affects binding to the canine transferrin receptor (TfR). The amino acid type at VP2 residues 93 and 323 has been shown to be the most important in controlling CPV-2 host range (Hueffer et al., 2003). CPV-2a strains from Uruguay also had distinctive amino acid substitutions in residue 324Ile; this substitution

Genetics and Molecular Research 16 (3): gmr16039719 
had been first reported in 2004 in CPV-2a strains from China and Korea (Pérez et al., 2012). The tyrosine (Tyr) residue is the most commonly found among the samples from CPV-2, followed by the isoleucine (Ile) residue in the position 324 of the VP2 gene. However, we have detected a CPV-2b strain with a different change at the position Tyr324Leu in all strains from Belém city, and it is the first reported mutation in this position of samples from Brazil and in the World. Further analyses are necessary to investigate the function of this mutation in the VP2 protein.

Phylogenetic analysis of the variants circulating in the canine population of some regions of Brazil has shown a great identity with other variants reported in the world; this fact confirms a cosmopolitan distribution of this virus (Pinto et al., 2012). However, phylogenetic analysis suggests that strains reported in this study were related to previously CPV-2a strains from Brazil, clustered with strains identified after 1990. These results suggest that strains from Belém city may have originated from CPV-2a circulated in Brazil between the 1990s and 2000s, although we need a complete sequence of CPV-2 from Brazil to confirm this hypothesis.

In addition to high rates and positive selection of mutations in the viral capsid gene, previous studies have reported that genetic recombination has an important factor in the generation of viral diversity and the emergence of new genotypes (Wang et al., 2012; Pérez et al., 2014). However, we not observed evidence about recombination events in strains from Belém reported in this study.

Continuous monitoring and molecular characterization of CPV-2 strains are needed to identify possible genetic and antigenic changes that may interfere with the effectiveness of vaccines, as well as provide a better understanding of the mechanisms that drive the evolution of CPV-2 in Brazil.

\section{Conflicts of interest}

The authors declare no conflict of interest.

\section{ACKNOWLEDGMENTS}

We are grateful for the economic support by PIBIC/IEC/CNPq/2015-2016 and Fundação de Amparo à Pesquisa do Estado de São Paulo, Brasil (Scholarships \#12/24150-9 and \#15/05778-5).

\section{REFERENCES}

Buonavoglia C, Martella V, Pratelli A, Tempesta M, et al. (2001). Evidence for evolution of canine parvovirus type 2 in Italy. J. Gen. Virol. 82: 3021-3025. https://doi.org/10.1099/0022-1317-82-12-3021

Cho H-S, Kang J-I and Park N-Y (2006). Detection of canine parvovirus in fecal samples using loop-mediated isothermal amplification. J. Vet. Diagn. Invest. 18: 81-84. https://doi.org/10.1177/104063870601800111

Cotmore SF, Agbandje-McKenna M, Chiorini JA, Mukha DV, et al. (2014). The family Parvoviridae. Arch. Virol. 159: 1239-1247. https://doi.org/10.1007/s00705-013-1914-1

Darriba D, Taboada GL, Doallo R and Posada D (2012). jModelTest 2: more models, new heuristics and parallel computing. Nat. Methods 9: 772-772. https://doi.org/10.1038/nmeth.2109

Decaro N, Desario C, Parisi A, Martella V, et al. (2009). Genetic analysis of canine parvovirus type 2c. Virology 385: 5-10. https://doi.org/10.1016/j.virol.2008.12.016

Fan X-X, Gao Y, Shu L, Wei Y-Q, et al. (2016). Transcriptome profiling indicating canine parvovirus type 2a as a potential immune activator. Virus Genes 52: 768-779. https://doi.org/10.1007/s11262-016-1363-5

Genetics and Molecular Research 16 (3): gmr16039719 
Felsenstein J (1985). Confidence limits on phylogenies: an approach using the bootstrap. Evolution 39: 783-791. https:// doi.org/10.1111/j.1558-5646.1985.tb00420.x

Hoelzer K, Shackelton LA, Parrish CR and Holmes EC (2008). Phylogenetic analysis reveals the emergence, evolution and dispersal of carnivore parvoviruses. J. Gen. Virol. 89: 2280-2289. https://doi.org/10.1099/vir.0.2008/002055-0

Hueffer K, Parker JSL, Weichert WS, Geisel RE, et al. (2003). The natural host range shift and subsequent evolution of canine parvovirus resulted from virus-specific binding to the canine transferrin receptor. J. Virol. 77: 1718-1726. https://doi.org/10.1128/JVI.77.3.1718-1726.2003

Huson DH and Bryant D (2006). Application of phylogenetic networks in evolutionary studies. Mol. Biol. Evol. 23: 254267. https://doi.org/10.1093/molbev/msj030

Katoh K and Standley DM (2013). MAFFT multiple sequence alignment software version 7: improvements in performance and usability. Mol. Biol. Evol. 30: 772-780. https://doi.org/10.1093/molbev/mst010

Margulies M, Egholm M, Altman WE, Attiya S, et al. (2005). Genome sequencing in microfabricated high-density picolitre reactors. Nature 437: 376-380.

Martin DP, Murrell B, Golden M, Khoosal A, et al. (2015). RDP4: Detection and analysis of recombination patterns in virus genomes. Virus Evol. 1: vev003. https://doi.org/10.1093/ve/vev003

Miranda C and Thompson G (2016). Canine parvovirus: the worldwide occurrence of antigenic variants. J. Gen. Virol. 97 : 2043-2057. https://doi.org/10.1099/jgv.0.000540

Myung IJ (2003). Tutorial on maximum likelihood estimation. J. Math. Psychol. 47: 90-100. https://doi.org/10.1016/ $\underline{\text { S0022-2496(02)00028-7 }}$

Parrish CR (1999). Host range relationships and the evolution of canine parvovirus. Vet. Microbiol. 69: 29-40. https://doi. org/10.1016/S0378-1135(99)00084-X

Parrish CR, Have P, Foreyt WJ, Evermann JF, et al. (1988). The global spread and replacement of canine parvovirus strains. J. Gen. Virol. 69: 1111-1116. https://doi.org/10.1099/0022-1317-69-5-1111

Parrish CR, Aquadro CF, Strassheim ML, Evermann JF, et al. (1991). Rapid antigenic-type replacement and DNA sequence evolution of canine parvovirus. J. Virol. 65: 6544-6552.

Pereira CA, Monezi TA, Mehnert DU, D'Angelo M, et al. (2000). Molecular characterization of canine parvovirus in Brazil by polymerase chain reaction assay. Vet. Microbiol. 75: 127-133. https://doi.org/10.1016/S0378-1135(00)00214-5

Pérez R, Bianchi P, Calleros L, Francia L, et al. (2012). Recent spreading of a divergent canine parvovirus type 2a (CPV-2a) strain in a CPV-2c homogenous population. Vet. Microbiol. 155: 214-219. https://doi.org/10.1016/j. vetmic.2011.09.017

Pérez R, Calleros L, Marandino A, Sarute N, et al. (2014). Phylogenetic and genome-wide deep-sequencing analyses of canine parvovirus reveal co-infection with field variants and emergence of a recent recombinant strain. PLoS One 9: e111779. https://doi.org/10.1371/journal.pone.0111779

Pinto LD, Streck AF, Gonçalves KR, Souza CK, et al. (2012). Typing of canine parvovirus strains circulating in Brazil between 2008 and 2010. Virus Res. 165: 29-33. https://doi.org/10.1016/j.virusres.2012.01.001

Pond SLK and Frost SDW (2005). Datamonkey: rapid detection of selective pressure on individual sites of codon alignments. Bioinformatics 21: 2531-2533. https://doi.org/10.1093/bioinformatics/bti320

Shackelton LA, Parrish CR, Truyen U and Holmes EC (2005). High rate of viral evolution associated with the emergence of carnivore parvovirus. Proc. Natl. Acad. Sci. USA 102: 379-384. https://doi.org/10.1073/pnas.0406765102

Stamatakis A (2014). RAxML version 8: a tool for phylogenetic analysis and post-analysis of large phylogenies. Bioinformatics 30: 1312-1313. https://doi.org/10.1093/bioinformatics/btu033

Strottmann DM, Scortegagna G, Kreutz LC, Barcellos LJG, et al. (2008). Diagnosis and serological study of canine parvovirus infection in dogs from Passo Fundo, Rio Grande do Sul, Brazil. Cienc. Rural 38: 400-405. https://doi. org $/ 10.1590 /$ S0103-84782008000200017

Wang J, Cheng S, Yi L, Cheng Y, et al. (2012). Evidence for natural recombination between mink enteritis virus and canine parvovirus. Virol. J. 9: 252. https://doi.org/10.1186/1743-422X-9-252

Zhang Z, Li J, Zhao X-Q, Wang J, et al. (2006). KaKs_Calculator: calculating Ka and Ks through model selection and model averaging. Genomics Proteomics Bioinformatics 4: 259-263. https://doi.org/10.1016/S1672-0229(07)60007-2

Genetics and Molecular Research 16 (3): gmr16039719 